\title{
Polytopal Approximation of Elongated Convex Bodies
}

\author{
Gilles Bonnet*
}

December 15, 2016

Primary 52A27, 52B11; Secondary 52B60.

\begin{abstract}
This paper presents bounds for the best approximation, with respect to the Hausdorff metric, of a convex body $K$ by a circumscribed polytope $P$ with a given number of facets. These bounds are of particular interest if $K$ is elongated. To measure the elongation of the convex set, its isoperimetric ratio $V_{j}(K)^{1 / j} V_{i}(K)^{-1 / i}$ is used.
\end{abstract}

Keywords. Polytopal approximation, elongated convex bodies, isoperimetric ratio, $\delta$-net.

MSC. Primary 52A27, 52B11; Secondary 52B60.

\section{Introduction and main results}

Let $K \subset \mathbb{R}^{d}$ be a full dimensional convex body, i.e. a compact convex set with non-empty interior. Fix $n \in \mathbb{N}$ and denote by $P=P_{K, n} \supset K$ a circumscribed polytope, with at most $n$ facets, minimizing the Hausdorff distance $d_{H}(K, P)$. It is well known that $d_{H}(K, P)$ is of order $n^{-2 /(d-1)}$ (see, e.g., [9]). Thus, we set $\mathfrak{d}_{1}(K, n):=d_{H}(K, P) n^{2 /(d-1)}$ and $\mathfrak{d}_{2}(K, n):=\sup _{m \geq n} \mathfrak{d}_{1}(K, m)$, and we have

$$
d_{H}(K, P)=\frac{\mathfrak{d}_{1}(K, n)}{n^{2 /(d-1)}} \leq \frac{\mathfrak{d}_{2}(K, n)}{n^{2 /(d-1)}} .
$$

Estimating $\mathfrak{d}_{1}(K, n)$ and $\mathfrak{d}_{2}(K, n)$ is a classical problem. We refer the reader to the well known surveys of P. M. Gruber [9, 12] and E. M. Bronstein [5] for an excellent overview of the huge amount of results and literature about polytopal approximation. The specificity of our main result is that we take into account how much $K$ is 'elongated'.

We denote by $V_{i}$ the $i$-th intrinsic volume (see Section 2 for the definition). For any $1 \leq i<j \leq d$, we call $V_{j}(K)^{1 / j} V_{i}(K)^{-1 / i}$ the $(i, j)$-isoperimetric

*gilles.bonnet@rub.de 
ratio of $K$. It is scale and translation invariant. The isoperimetric inequality (see Section 2, inequality (2), for a statement) says that it is maximized by the balls. On the other hand, $V_{j}(K)^{1 / j} V_{i}(K)^{-1 / i} \simeq 0$ precisely when the normalized body $V_{i}(K)^{-1 / i} K$ is close to a $(j-1)$-dimensional convex body. If an isoperimetric ratio of $K$ is close to zero, we say that $K$ is elongated. More precisely, if $V_{j}(K)^{1 / j} V_{i}(K)^{-1 / i}<\epsilon$, we say that $K$ is $(\epsilon: i, j)$-elongated. The following theorem gives a bound for the Hausdorff distance between a convex body $K$ and its best approximating polytope. This bound can be arbitrarily small if $K$ is sufficiently elongated.

Theorem 1.1. Assume $1 \leq i<j \leq\lceil(d-1) / 2\rceil$. Set $\alpha=2\lceil(d-1) / 2\rceil(d-$ 1) $d^{-1}$ and $\beta=\lceil(d-1) / 2\rceil(d-1)^{-1} d^{-1}$. There exist constants $\delta_{i, j}$ and $n_{i, j}$, both depending on $d$, such that the following holds. For any $\epsilon>0$ and any convex body $K$

$$
\text { if } K \text { is }(\epsilon: i, j) \text {-elongated then } \mathfrak{d}_{2}\left(K, n_{i, j} \epsilon^{-\alpha}\right)<\delta_{i, j} \epsilon^{\beta} V_{1}(K) \text {. }
$$

I.e. for any $\epsilon>0$ and any convex body $K$

$$
\text { if } \frac{V_{j}(K)^{1 / j}}{V_{i}(K)^{1 / i}}<\epsilon \text { then } d_{H}(K, P)<\delta_{i, j} \epsilon^{\beta} \frac{V_{1}(K)}{n^{2 /(d-1)}} \text { for any } n \geq n_{i, j} \epsilon^{-\alpha},
$$

where $P=P_{K, n} \supset K$ is a circumscribed polytope, with at most $n$ facets, minimizing the Hausdorff distance $d_{H}(K, P)$.

Note that the case $i=1$ and $j=\lceil(d-1) / 2\rceil$ implies all the others. This is a consequence of the isoperimetric inequality. We conjecture that Theorem 1.1 remains true for any $1 \leq i<j \leq d-1$ and that $\beta$ could be replaced by 1 . Equation (1) below gives support to this conjecture.

Let us recall a few important results in order to motivate Theorem 1.1. If $K$ has a twice differentiable smooth boundary, we have a precise asymptotic approximation of $\mathfrak{d}_{2}(K, n)$. After planar results due to Tóth [8] and McClure and Vitale [14], Schneider [17, 18] and Gruber [10] succeeded in proving that

$$
\lim _{n \rightarrow \infty} \mathfrak{d}_{2}(K, n)=\frac{1}{2}\left(\frac{\vartheta_{d-1}}{\kappa_{d-1}} \int_{\partial K} \kappa_{C}(\boldsymbol{x})^{1 / 2} \sigma(\mathrm{d} \boldsymbol{x})\right)^{2 /(d-1)},
$$

where $\vartheta_{k}$ is the minimum covering density of $\mathbb{R}^{k}$ with balls of fixed radius, $\kappa_{k}$ the volume of the $k$-dimensional ball, $\kappa_{C}(\boldsymbol{x})>0$ the Gaussian curvature of $K$ at the point $\boldsymbol{x}$, and $\sigma(\cdot)$ the surface area measure. More recently, Böröczky [3] removed the condition $\kappa_{C}(\boldsymbol{x})>0$.

In many practical situations it is out of reach to compute the integral explicitly. But if $K$ is elongated, we can have a good upper bound. Hölder's inequality implies

$$
\begin{aligned}
\int_{\partial K} \kappa_{C}(\boldsymbol{x})^{1 / 2} \mathrm{~d} \sigma(\boldsymbol{x}) & \leq\left(2 d \kappa_{d}\right)^{1 / 2} V_{d-1}(K)^{1 / 2} \\
& =\left(2 d \kappa_{d}\right)^{1 / 2}\left[\frac{V_{d-1}(K)^{1 /(d-1)}}{V_{1}(K)}\right]^{(d-1) / 2} V_{1}(K)^{(d-1) / 2} .
\end{aligned}
$$


Hence, with the isoperimetric inequality, for any $1 \leq i<j \leq d-1$, we have that

$$
\text { if } K \text { is }(\epsilon: i, j) \text {-elongated then } \lim _{n \rightarrow \infty} \mathfrak{d}_{2}(K, n) \leq \delta_{i, j}^{\prime} \epsilon V_{1}(K)
$$

with

$$
\delta_{i, j}^{\prime}:=\frac{1}{2}\left(\frac{\vartheta_{d-1}}{\kappa_{d-1}}\right)^{2 / d-1}\left(2 d \kappa_{d}\right)^{1 /(d-1)} \frac{V_{d-1}\left(B^{d}\right)^{1 /(d-1)}}{V_{1}\left(B^{d}\right)} \frac{V_{i}\left(B^{d}\right)^{1 / i}}{V_{j}\left(B^{d}\right)^{1 / j}} .
$$

Therefore, we have a good asymptotic bound for elongated smooth convex bodies.

The main goal of this paper is to extend these results to the non-asymptotic and non-smooth case. The order $\epsilon$ in (1) should be compared to the order $\epsilon^{\beta}$ in Theorem 1.1 for a fixed $n$. It is especially of interest, for example, if we approximate a polytope with many facets by one with fewer facets. This was considered by Reisner, Schütt and Werner in [15]. This paper was the starting point of our investigations. A reader who has studied it will notice that principal ideas of their work are still present in our proofs.

Theorem 1.1 should be compared to the following result. It was obtained independently in [6] and [7]. The constants were improved in [15]. There exist constants $c_{1}(d)$ and $c_{2}(d)$ such that $\mathfrak{d}_{2}\left(K, c_{2}(d)\right)<c_{1}(d) R(K)$, i.e.

$$
d_{H}(K, P) \leq c_{1}(d) \frac{R(K)}{n^{2 /(d-1)}} \text { for } n>c_{2}(d),
$$

where $R(K)$ is the radius of the smallest ball containing $K$. Note that $R(K)$ is of the same order as $V_{1}(K)$. Although this bound is sharp in general, it is worse if we assume that $K$ is elongated. The following is an example of such a situation. Fix a small $\epsilon>0$. Let $K \subset \mathbb{R}^{4}$ be a convex body. It is well known that there exists an ellipsoid $E$ such that $E \subset K \subset d E$ (see, e.g., [13]). Let $r_{1}>\cdots>r_{4}$ be the lengths of the principal axes of $E$. Assume that $r_{1}=1$ and $r_{2}$ is sufficiently small. Thus, $K$ is $(\epsilon: 1,2)$-elongated. For $n>n_{1,2} \epsilon^{-3}$, Theorem 1.1 says that $\mathfrak{d}_{1}(K, n)<\delta_{1,2} \epsilon^{1 / 6} \ll c_{1}(d) R(K)$.

Finally, we would like to highlight the following theorem. Not only is it an important step in the proof of Theorem 1.1 but also an interesting result on its own.

Theorem 1.2. There exist absolute constants $\boldsymbol{c}_{1}$ and $\boldsymbol{c}_{2}$, independent of $d$, such that the following holds. Let $K$ be a convex body. Then,

$$
\mathfrak{d}_{2}\left(K, \boldsymbol{c}_{1}^{d} d^{d / 2} V_{d-1}\left(K+B^{d}\right)\right)<\boldsymbol{c}_{2} d V_{d-1}\left(K+B^{d}\right)^{2 /(d-1)} .
$$

I.e. for any integer $n>c_{1}^{d} d^{d / 2} V_{d-1}\left(K+B^{d}\right)$, there exists a polytope $P \supset K$ with $n$ facets such that

$$
\mathrm{d}_{H}(K, P)<\boldsymbol{c}_{2} d V_{d-1}\left(K+B^{d}\right)^{2 /(d-1)} n^{-2 /(d-1)} .
$$


The paper is structured as follows. In the next section setting, notation and background material from convex geometry are provided. In Section 3 we give a general framework to build a $\delta$-net on an abstract measured metric space satisfying mild properties, and we apply it to prove Theorem 1.2. The proof of Theorem 1.1 is given in Section 5. It uses a shape factor introduced and described in Section 4.

\section{Setting, notation and background}

We work in the euclidean space $\mathbb{R}^{d}$ with origin $\boldsymbol{o}$, scalar product $\langle\cdot, \cdot\rangle$ and associated norm $|\cdot|$. We denote by $B(\boldsymbol{x}, r)$ and $S(\boldsymbol{x}, r)$, respectively, the ball and the sphere of center $\boldsymbol{x}$ and radius $r$. The unit ball $B^{d}=B(\boldsymbol{o}, 1)$ has volume $\kappa_{d}$ and the unit sphere $\mathbb{S}^{d-1}=S(\boldsymbol{o}, 1)$ has surface area $\omega_{d}=d \kappa_{d}$. We denote by $\mathcal{K}$ the set of convex bodies (compact and convex sets of $\mathbb{R}^{d}$ ) with at least 2 points. This set is equipped with the Hausdorff distance

$$
d_{H}(K, L)=\min _{r \geq 0}\left(K \subset L+r B^{d}, L \subset K+r B^{d}\right)
$$

and its associated topology and Borel structure. The same holds for the future subsets of $\mathcal{K}$ that we will encounter in this paper. The set $\mathcal{K}$ is also equipped with Minkowski sum and scale action. For any $t \in \mathbb{R}$ and $A, B \in \mathcal{K}$, we have

$$
t A:=\{t \boldsymbol{a}: \boldsymbol{a} \in A\}, \quad A+B:=\{\boldsymbol{a}+\boldsymbol{b}: \boldsymbol{a} \in A, \boldsymbol{b} \in B\} .
$$

We denote by $\partial K$ the boundary of a given convex body $K$.

Let $f: \mathcal{K} \rightarrow \mathbb{R}$ be a map. If there exists $k \in \mathbb{R}$ such that $f(t K)=t^{k} K$ for any $K \in \mathcal{K}$ and $t>0$, we say that $f$ is homogeneous of degree $k$. We say that $f$ is scale invariant if $f$ is homogeneous of degree 0 . If $f(K+\boldsymbol{x})=f(K)$ for any $K \in \mathcal{K}$ and $\boldsymbol{x} \in \mathbb{R}^{d}$, we say that $f$ is translation invariant. We say that $f$ is a shape factor if $f$ is scale and translation invariant.

For the following facts of convex geometry we refer the reader to [11].

The Steiner Formula and Intrinsic Volumes. We denote by $V_{d}(\cdot)$ the volume, i.e., the $d$-dimensional Lebesgue measure. The Steiner Formula tells us that there exist functionals $V_{i}: \mathcal{K} \rightarrow[0, \infty)$, for $0 \leq i \leq d$, such that for any $K \in \mathcal{K}$ and $\epsilon \geq 0$

$$
V_{d}\left(K+\epsilon B^{d}\right)=\sum_{i=0}^{d} \epsilon^{d-j} \kappa_{d-j} V_{j}(K) .
$$

$V_{i}(K)$ is called the $i$-th intrinsic volume of $K$. Some of the intrinsic volumes have a clear geometric meaning. $V_{d}$ is the volume. If $K$ has non-empty interior, then

$$
V_{d-1}(K)=\frac{1}{2} \mathcal{H}^{d-1}(\partial K),
$$

where $\mathcal{H}^{d-1}(\partial K)$ is the $(d-1)$-dimensional Hausdorff measure of the boundary $\partial K$ of $K$. Thus, $2 V_{d-1}$ is the surface area. $V_{1}$ is proportional to the mean 
width $b$. More precisely,

$$
\frac{d \kappa_{d}}{2} b(K)=\kappa_{d-1} V_{1}(K)=\int_{\mathbb{S}^{d-1}} h(K, \boldsymbol{u}) \sigma(\mathrm{d} \boldsymbol{u}),
$$

where $\sigma$ is the surface area measure on $\mathbb{S}^{d-1}$ and $h(K, \boldsymbol{u}):=\max \{\langle\boldsymbol{x}, \boldsymbol{u}\rangle$ : $\boldsymbol{x} \in K\}$ is the value of the support function of $K$ at $\boldsymbol{u} . V_{0}(K)=1$ is the Euler characteristic. For $1 \leq i<j \leq d$ and $K \in \mathcal{K}$, we call the shape factor $V_{j}(K)^{1 / j} V_{i}(K)^{-1 / i}$ the $(i, j)$-isoperimetric ratio of $K$.

The Isoperimetric Inequality. Let $B \subset \mathbb{R}^{d}$ be a $d$-dimensional ball. For any $K \in \mathcal{K}$ and for any $1 \leq i<j \leq d$,

$$
V_{j}(K)^{1 / j} \leq \frac{V_{j}(B)^{1 / j}}{V_{i}(B)^{1 / i}} V_{i}(K)^{1 / i},
$$

with equality if and only if $K$ is a ball.

A Steiner-type Formula. For any $K \in \mathcal{K}$

$$
V_{d-1}\left(K+B^{d}\right)=\sum_{k=0}^{d-1} \frac{(d-k) \kappa_{d-k}}{2 d} V_{k}(K) .
$$

The isoperimetric inequality and the Steiner-type formula imply the next fact.

Fact : Let $d \geq 3, I$ be an interval (convex hull of two distinct points), $B$ be a ball, and $K \in \mathcal{K}$ neither an interval nor a ball. Assume that $V_{1}(I)=$ $V_{1}(K)=V_{1}(B)$. Note that $V_{1}(I)$ is just the length of the segment $I$. Then, we have

$$
V_{d-1}\left(I+B^{d}\right)<V_{d-1}\left(K+B^{d}\right)<V_{d-1}\left(B+B^{d}\right) .
$$

Convention about the constants. The constants are denoted by $c_{i}$, where $i$ is an index. They depend on $d$ but are independent of any other quantity. We estimate the dependence on $d$ using the Landau notation. By $c_{i}=\boldsymbol{\Theta}(f(d))$ we mean that there exist absolute constants $k_{0}, k_{1}>0$ such that $k_{0} f(d)<c_{i}<k_{1} f(d)$ for any $d$.

\section{$3 \quad \delta$-net and polytopal approximation}

First, let us set some notation. Assume $M$ is metric space with distance $d_{M}$. I.e. a set $M$ and a function $d_{M}: M \times M \rightarrow[0, \infty)$ such that, for any $x, y, z \in M, d_{M}(x, y)=0$ if and only if $x=y, d_{M}(x, y)=d_{M}(y, x)$, and $d_{M}(x, z) \leq d_{M}(x, y)+d_{M}(y, z)$. We write $B_{M}(\boldsymbol{x}, r):=\left\{\boldsymbol{y} \in M: d_{M}(\boldsymbol{x}, \boldsymbol{y}) \leq\right.$ $r\}$.

Definition 3.1. Let $M$ be a metric space and $S$ a discrete subset of $M$. We say that

- $S$ is a $\delta$-covering of $M$ if $\cup_{\boldsymbol{x} \in S} B_{M}(\boldsymbol{x}, \delta)=M$,

- $S$ is a $\delta$-packing of $M$ if $B_{M}(\boldsymbol{x}, \delta) \cap B_{M}(\boldsymbol{y}, \delta)=\emptyset$ for any $\boldsymbol{x} \neq \boldsymbol{y} \in S$, 
- $S$ is a $\delta$-net of $M$ if it is both a $\delta$-covering of $M$ and a $(\delta / 2)$-packing of $M$.

Note that, in the poset of $(\delta / 2)$-packings ordered under inclusion, a maximal element is a $\delta$-net. Zorn's lemma shows that, for any metric space $M$, there exists a $\delta$-net.

In the following lemma, under some assumptions on $\psi$, we give bounds for the cardinality of a $\delta$-net. The construction of these bounds is adapted from the proof of the following well known result, see e.g. [11, Proposition 31.1]. If $C \subset \mathbb{R}^{d}$ is a convex body with non empty interior such that $C=-C$, then there exists a packing of translated copies of $C$ in $\mathbb{R}^{d}$ of density at least $2^{-d}$, where, roughly speaking, density means the proportion of $\mathbb{R}^{d}$ covered by the translated copies of $C$.

Lemma 3.2. Let $M$ be a space equipped with a measure $\psi$ and a measurable metric $d_{M}$. Assume that $\psi(M)<\infty$. Let $\delta_{0}>0$ and $S$ be a $\delta$-net of $M$ with $\delta \in\left(0, \delta_{0}\right)$. Let $k>0$.

1. Assume there exists a constant $c>0$ such that, for any $\boldsymbol{x} \in M$ and $r \in\left(0, \delta_{0}\right)$, it holds that $c r^{k}>\psi\left(B_{M}(\boldsymbol{x}, r)\right)$. Then $|S|>c^{-1} \psi(M) \delta^{-k}$.

2. Assume there exists a constant $c^{\prime}>0$ such that, for any $\boldsymbol{x} \in M$ and $r \in$ $\left(0, \delta_{0}\right)$, it holds that $c^{\prime} r^{k}<\psi\left(B_{M}(\boldsymbol{x}, r)\right)$. Then $|S|<2^{k} c^{\prime-1} \psi(M) \delta^{-k}$.

Proof. To prove (1), we only have to observe that since $S$ is a $\delta$-covering, we have that

$$
\psi(M) \leq \sum_{\boldsymbol{x} \in S} \psi\left(B_{M}(\boldsymbol{x}, \delta)\right)<|S| c \delta^{k}
$$

because $M=\cup_{\boldsymbol{x} \in S} B_{M}(\boldsymbol{x}, \delta)$. The proof of (2) is similar. Since $S$ is a $(\delta / 2)$ packing, we have that

$$
\psi(M) \geq \sum_{\boldsymbol{x} \in S} \psi\left(B_{M}(\boldsymbol{x}, \delta / 2)\right)>|S| c^{\prime} \delta^{k} 2^{-k}
$$

because, for any distinct $\boldsymbol{x}, \boldsymbol{y} \in S$, we have $B_{M}(\boldsymbol{x}, \delta / 2) \cap B_{M}(\boldsymbol{y}, \delta / 2)=\emptyset$.

In Lemma 3.4, we will apply the previous lemma to the space $M=\partial(K+$ $\left.B^{d}\right)$, where $K$ is an arbitrary convex body and $M$ is equipped with the surface area measure and the restriction of the euclidean distance. In this space the balls are caps on the boundary of the convex body $D=K+B^{d}$, where a cap is defined as follow. For a convex body $D$, a point $\boldsymbol{d} \in D$ (usually $\boldsymbol{d} \in \partial D$ ), and a positive radius $\delta>0$, we define the cap of $D$ of center $\boldsymbol{d}$ and radius $\delta$ to be the set

$$
\operatorname{cap}(D, \boldsymbol{d}, \delta)=\{\boldsymbol{y} \in \partial D:|\boldsymbol{d}-\boldsymbol{y}|<\delta\} .
$$

Note that our definition differs slightly from the more usual one, where a cap is the intersection of the boundary $\partial D$ with a half-space. In the next lemma we give bounds for the surface area of caps of radius $\delta \in\left(0, \delta_{0}\right)$, of bodies of the form $K+B^{d}$, with $\delta_{0}=1$ independent from $K$. Precise bounds for spherical caps are known, see e.g. Lemma 2.1 in [4], Lemmas 2.2 and 2.3 in 


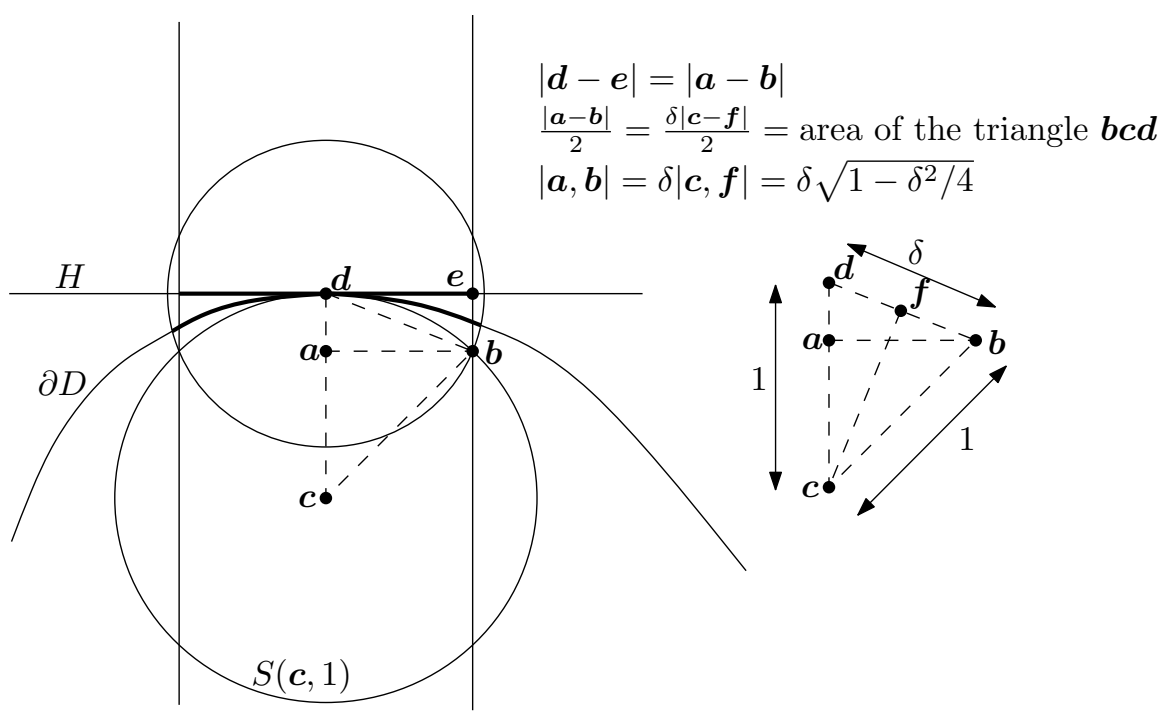

Figure 1: $\quad \mathcal{H}^{d-1}(\operatorname{cap}(D, \boldsymbol{d}, \delta)) \geq \delta^{d-1} \kappa_{d-1}\left(1-\frac{\delta^{2}}{4}\right)^{(d-1) / 2}$

[2] or Remark 3.1.8 in [1]. Lemma 6.2 in [16] gives bounds for more general bodies then the sphere, namely those with $\mathcal{C}^{2}$ boundary of positive curvature, but with a $\delta_{0}$ depending on $K$. It does not seems to the author that we can deduce easily Lemma 3.3 from these results.

Lemma 3.3. Let $K \in \mathcal{K}$ and $D=K+B^{d}$. Let $\boldsymbol{d} \in \partial D$ and $\delta \in(0,1)$. Then

$$
\delta^{d-1} \kappa_{d-1} 2^{-(d-1)}<\mathcal{H}^{d-1}(\operatorname{cap}(D, \boldsymbol{d}, \delta))<\delta^{d-1} \kappa_{d-1} d .
$$

Proof. For the lower bound, we approximate the cap by a $(d-1)$-dimensional disc of radius $\delta \sqrt{1-\delta^{2} / 4}$ (see Figure 1). Let $H$ be the tangent hyperplane to $D$ at $\boldsymbol{d}$. We have

$$
\begin{aligned}
\mathcal{H}^{d-1}(\operatorname{cap}(D, \boldsymbol{d}, \delta)) & \geq \mathcal{H}^{d-1}(H \cap B(\boldsymbol{d}, d(\boldsymbol{d}, \boldsymbol{e}))) \\
& =\delta^{d-1} \kappa_{d-1}\left(1-\frac{\delta^{2}}{4}\right)^{(d-1) / 2} \\
& >\delta^{d-1} \kappa_{d-1}\left(\frac{3}{4}\right)^{(d-1) / 2}>\delta^{d-1} \kappa_{d-1} 2^{-(d-1)} .
\end{aligned}
$$

For the upper bound, we approximate the cap by the union of a $(d-1)$ dimensional disc of radius $\delta$ and the spherical boundary of a cylinder of radius $\delta$ and height $\delta^{2}$ (see Figure 2). Thus

$$
\begin{aligned}
\mathcal{H}^{d-1}(\operatorname{cap}(D, \boldsymbol{d}, \delta)) & <\mathcal{H}^{d-1}(H \cap B(\boldsymbol{d}, \delta))+\mathcal{H}^{d-2}(H \cap S(\boldsymbol{d}, \delta)) \delta^{2} \\
& =\delta^{d-1} \kappa_{d-1}+\delta^{d-2} \omega_{d-1} \delta^{2} \\
& =\delta^{d-1} \kappa_{d-1}(1+\delta(d-1))<\delta^{d-1} \kappa_{d-1} d .
\end{aligned}
$$




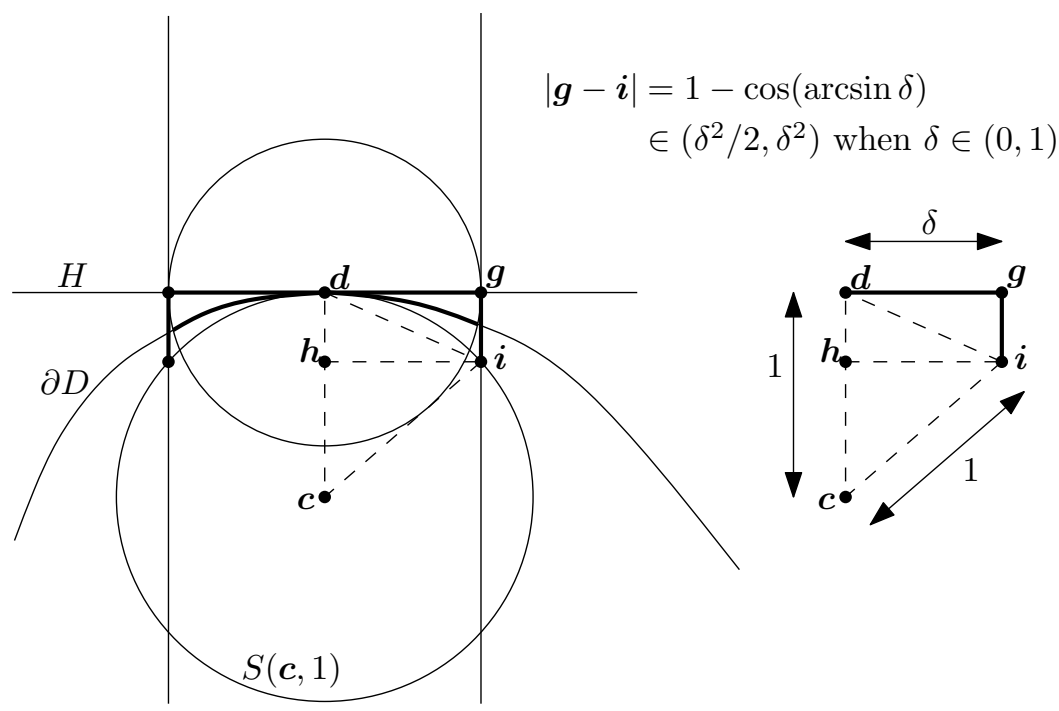

Figure 2: $\quad \mathcal{H}^{d-1}(\operatorname{cap}(D, \boldsymbol{d}, \delta))<\delta^{d-1} \kappa_{d-1}+\delta^{d-2} \omega_{d-1} \delta^{2}$

Set $c_{3}:=2 d^{-1} \kappa_{d-1}^{-1}=\boldsymbol{\Theta}\left(d^{1 / 2}\right)^{d}$ and $c_{4}:=4^{d} \kappa_{d-1}^{-1}=\boldsymbol{\Theta}\left(d^{1 / 2}\right)^{d}$. As a direct consequence of the two previous lemmas and the fact that $\mathcal{H}^{d-1}(\partial D)=$ $2 V_{d-1}(D)$, we have the following lemma. We omit the proof.

Lemma 3.4. Let $K \in \mathcal{K}$ and $D=K+B^{d}, \delta \in(0,1)$ and $S$ a $\delta$-net of the boundary $\partial D$. We have that

$$
c_{3} V_{d-1}(D) \delta^{-(d-1)}<|S|<c_{4} V_{d-1}(D) \delta^{-(d-1)} .
$$

For a convex body $K$ with boundary $\partial K$ of differential class $\mathscr{C}^{1}$ and $\boldsymbol{x} \in$ $\partial K$, we denote by $\boldsymbol{v}(\boldsymbol{x})$ the outer unit normal vector of $K$ at $\boldsymbol{x}$. Using Lemma 3.4 , we can prove the two following lemmas in a similar way as Propositions 2.4 and Proposition 2.7 of [15]. We will only sketch the proofs.

Lemma 3.5. Let $K \in \mathcal{K}$ with $\partial K$ of class $\mathscr{C}^{1}$ and $\delta \in(0,1)$. There exists a $\delta$ net of $\partial K$, with respect to the distance $d_{m}(\boldsymbol{x}, \boldsymbol{y})=\max (|\boldsymbol{x}-\boldsymbol{y}|,|\boldsymbol{v}(\boldsymbol{x})-\boldsymbol{v}(\boldsymbol{y})|)$, of cardinality at most $c_{4} V_{d-1}\left(K+B^{d}\right) \delta^{-(d-1)}$.

Sketch of the proof. Set $D=K+B^{d}$. Construct a $\delta$-net on the boundary $\partial D$ and then project it onto $\partial K$. The bound on the cardinality comes from Lemma 3.4.

Set $c_{5}:=3^{(d-1) / 4} c_{4}=\boldsymbol{\Theta}\left(d^{1 / 2}\right)^{d}$.

Lemma 3.6. Let $K \in \mathcal{K}$ and $0<\epsilon<1$. Then, there exists a polytope $P_{\epsilon} \supset K$ with

$$
\mathrm{d}_{H}\left(K, P_{\epsilon}\right)<\epsilon
$$

and with number of facets at most

$$
c_{5} V_{d-1}\left(K+B^{d}\right) \epsilon^{-(d-1) / 2} .
$$


Sketch of the proof. Reduce the proof to the case where $K$ has a smooth boundary. Set an appropriate value $\delta=\delta(\epsilon)$. Consider the $\delta$-net $S$ built in Lemma 3.5. Construct the circumscribed polytope $P \supset C$ with one facet tangent to $C$ at each point of $S$. Finally bound the Hausdorff distance $d_{H}(C, P)$. The bound on the number of facets comes from the bound on the cardinality of the $\delta$-net in Lemma 3.5.

Set $c_{6}:=c_{5}^{2 /(d-1)}=\boldsymbol{\Theta}(d)$. With the last lemma, we can now prove Theorem 1.2.

Proof of Theorem 1.2. Let $n>c_{5} V_{d-1}\left(K+B^{d}\right)$.

Set $\epsilon=c_{6} V_{d-1}\left(K+B^{d}\right)^{2 /(d-1)} n^{-2 /(d-1)}$. By the assumption made on $n$, we have $\epsilon<1$. Hence, we can apply Lemma 3.6. There exists a polytope $P_{\epsilon} \supset K$ with $d_{H}\left(K, P_{\epsilon}\right)<\epsilon$ and such that its number of facets is at most

$$
c_{5} V_{d-1}\left(K+B^{d}\right) \epsilon^{-(d-1) / 2}=n .
$$

The approximations of the constants $c_{i}$ using the Landau notation tells us that there exist absolute constants $\boldsymbol{c}_{1}$ and $\boldsymbol{c}_{2}$ such that $c_{5}<\boldsymbol{c}_{1}^{d} d^{d / 2}$ and $c_{6}<\boldsymbol{c}_{2} d$ for any $d$. This yields the proof.

\section{Shape factor}

In this section we define $\mathfrak{g}_{l}$, a shape factor, i.e. a scale and translation invariant function on $\mathcal{K}$. Lemma 4.2 tells us how $\mathfrak{g}_{l}(K)$ describes the elongation of a given convex body $K$.

Set $c_{7}:=c_{5} V_{d-1}\left(B^{d}\right)$.

Definition 4.1. For any fixed parameter $l>c_{7}$ we define the functions $\mathfrak{b}_{l}, \mathfrak{f}_{l}, \mathfrak{g}_{l}: \mathcal{K} \rightarrow(0, \infty)$ by

$$
\begin{gathered}
\mathfrak{b}_{l}(K)=\sup \left\{t \in(0, \infty): l>c_{5} V_{d-1}\left(t K+B^{d}\right)\right\}, \\
\mathfrak{f}_{l}(K)=\inf _{t \in\left(0, \mathfrak{b}_{l}(K)\right)} \frac{V_{d-1}\left(t K+B^{d}\right)^{2 /(d-1)}}{t},
\end{gathered}
$$

and

$$
\mathfrak{g}_{l}(K)=\frac{\mathfrak{f}_{l}(K)}{V_{1}(K)} .
$$

It is clear that the three functions are translation invariant. One can check that $\mathfrak{b}_{l}$ is homogeneous of degree $-1, \mathfrak{f}_{l}$ is homogeneous of degree 1 and $\mathfrak{g}_{l}$ is homogeneous of degree 0 . Therefore, for any fixed $l, \mathfrak{g}_{l}$ is a shape factor. The next lemma gives a geometric interpretation of $\mathfrak{g}_{l}$.

\section{Lemma 4.2.}

1. For any $K \in \mathcal{K}$, the function $l \mapsto \mathfrak{g}_{l}(K)$ is decreasing.

2. If $d=2$ and $l>c_{7}$ is fixed, then $\mathfrak{g}_{l}$ is constant on $\mathcal{K}$. 
3. If $d \geq 3, l>c_{7}$ is fixed, and $K \in \mathcal{K}$ is neither an interval nor a ball, then

$$
\mathfrak{g}_{l}(I)<\mathfrak{g}_{l}(K)<\mathfrak{g}_{l}(B) \text { for any } l>c_{7},
$$

where $I$ denotes an interval and $B$ a ball.

4. Assume that $1 \leq i<j \leq\lceil(d-1) / 2\rceil$. There exist constants $\delta_{i, j}$ and $n_{i, j}$, both depending on $d$, such that the following holds. For any convex body $K \in \mathcal{K}$ and $\epsilon>0$, we have

$$
\text { if } \frac{V_{j}(K)^{1 / j}}{V_{i}(K)^{1 / i}}<\epsilon \text { then } \mathfrak{g}_{N_{i, j}(\epsilon)}(K) \leq \delta_{i, j} \epsilon^{\beta},
$$

where $N_{i, j}(\epsilon):=n_{i, j} \epsilon^{-\alpha}$ with $\alpha=2\lceil(d-1) / 2\rceil(d-1) d^{-1}$, and $\beta=$ $2\lceil(d-1) / 2\rceil(d-1)^{-1} d^{-1}$.

Proof. (1) is a direct consequence of the definition of $\mathfrak{g}_{l}$. (2) comes from the fact that in this case $V_{d-1}=V_{1}$ is additive. (3) is implied by (4). It only remains to prove (4).

For the rest of the proof we write $v_{i}:=V_{i}\left(B^{d}\right)^{1 / i}$ for $i=1, \ldots, d$. Thanks to point 3 of the present lemma, we have that $\mathfrak{g}_{N_{i, j}(\epsilon)}(K) \leq \mathfrak{g}_{N_{i, j}(\epsilon)}(B)$. This implies that, without loss of generality, we can assume that $\epsilon\langle c$, for $c>0$ as small as one need. We also reduce the proof to the case $i=1$ and $j=j_{0}=$ $\lceil(d-1) / 2\rceil$. Because of the isoperimetric inequality (2), we have

$$
\frac{V_{j_{0}}(K)^{1 / j_{0}}}{V_{1}(K)} \leq c_{i, j} \frac{V_{j}(K)^{1 / j}}{V_{i}(K)^{1 / i}} \text { where } c_{i, j}:=\frac{v_{j_{0}} v_{i}}{v_{j} v_{1}} .
$$

Assume that there exist constants $\delta_{1, j_{0}}$ and $n_{1, j_{0}}$ such that (5) holds for $i=1$ and $j=j_{0}$. Let $1 \leq i<j \leq j_{0}$ and $(i, j) \neq\left(1, j_{0}\right)$. We set $\delta_{i, j}:=\delta_{1, j_{0}} c_{i, j}^{\beta}$ and $n_{i, j}:=n_{1, j_{0}} c_{i, j}^{-\alpha}$. In particular, $N_{i, j}(\epsilon)=n_{i, j} \epsilon^{-\alpha}=$ $n_{1, j_{0}}\left(c_{i, j} \epsilon\right)^{-\alpha}=N_{1, j_{0}}\left(c_{i, j} \epsilon\right)$. Assume that $K$ is such that $V_{j}(K)^{1 / j} V_{i}(K)^{-1 / i}<$ $\epsilon$. By (6) we have $V_{j_{0}}(K)^{1 / j_{0}} V_{1}(K)<c_{i, j} \epsilon$. This implies that $\mathfrak{g}_{N_{i, j}(\epsilon)}(K)=$ $\mathfrak{g}_{N_{1, j_{0}}\left(c_{i, j} \epsilon\right)}(K) \leq \delta_{1, j_{0}}\left(c_{i, j} \epsilon\right)^{\beta}=\delta_{i, j} \epsilon^{\beta}$. This shows that we only have to consider the case $i=1$ and $j=j_{0}$.

Since both parts of (5) are scale invariant, we also assume without loss of generalities that $V_{1}(K)=1$. Let $\epsilon \in(0,1)$ and $l>c_{7}$. From now on, we assume that

$$
V_{j_{0}}(K)^{1 / j_{0}}<\epsilon .
$$

Set

$$
p_{C}(t):=V_{d-1}\left(t K+B^{d}\right) \stackrel{(3)}{=} \sum_{k=0}^{d-1} \frac{(d-k) \kappa_{d-k}}{2 d} V_{k}(K) t^{k} .
$$

Observe that it is a strictly increasing and continuous function and that

$$
\begin{aligned}
\mathfrak{g}_{l}(K) & =\mathfrak{f}_{l}(K)=\left(\inf _{t \in\left(0, \mathfrak{b}_{l}(K)\right)} t^{-(d-1) / 2} p_{C}(t)\right)^{2 /(d-1)} \\
\text { and } \mathfrak{b}_{l}(K) & =p_{C}^{-1}\left(c_{5}^{-1} l\right) \text {. }
\end{aligned}
$$


Observe that $j_{0}-1-(d-1) / 2 \leq-1 / 2$. Hence, for $t>1$,

$$
t^{-(d-1) / 2} p_{C}(t) \leq S_{1}(K) t^{-1 / 2}+S_{2}(K) t^{(d-1) / 2},
$$

where

$$
S_{1}(K):=\sum_{k=0}^{j_{0}-1} \frac{(d-k) \kappa_{d-k}}{2 d} V_{k}(K) \text { and } S_{2}(K):=\sum_{k=j_{0}}^{d-1} \frac{(d-k) \kappa_{d-k}}{2 d} V_{k}(K) .
$$

The isoperimetric inequalities (2) gives that

$$
S_{1}(K) \leq \frac{\kappa_{d}}{2}+\sum_{k=1}^{j_{0}-1} \frac{(d-k) \kappa_{d-k}}{2 d}\left(\frac{v_{k}}{v_{1}}\right)^{k}=: c_{8} .
$$

It also implies that, for $k=j_{0}, \ldots, d-1$, we have $V_{k}(K) \leq\left(v_{k} / v_{j_{0}}\right)^{k} V_{j_{0}}(K)^{k / j_{0}}$. And since $V_{j_{0}}(K)^{k / j_{0}}<\epsilon^{k} \leq \epsilon^{j_{0}}$, it follows that

$$
S_{2}(K) \leq \sum_{k=j_{0}}^{d-1} \frac{(d-k) \kappa_{d-k}}{2 d}\left(\frac{v_{k}}{v_{j_{0}}}\right)^{k} \epsilon^{j_{0}}=: c_{9} \epsilon^{j_{0}} .
$$

Therefore, for $t>1$,

$$
t^{-(d-1) / 2} p_{C}(t) \leq c_{8} t^{-1 / 2}+c_{9} \epsilon^{j_{0}} t^{(d-1) / 2}=: q_{\epsilon}(t) .
$$

Since we want $t^{-(d-1) / 2} p_{C}(t)$ small, we define $t_{\epsilon}>0$ such that $q_{\epsilon}\left(t_{\epsilon}\right)$ is minimal. But it holds that the derivative of $q_{\epsilon}$ is

$$
q_{\epsilon}^{\prime}(t)=\frac{-c_{8}}{2} t^{-3 / 2}+\frac{c_{9} \epsilon^{j_{0}}(d-1)}{2} t^{(d-3) / 2} .
$$

Thus,

$$
t_{\epsilon}=\left(\frac{c_{9} \epsilon^{j_{0}}(d-1)}{c_{8}}\right)^{-2 / d}=c_{10} \epsilon^{-2 j_{0} / d}
$$

with $c_{10}:=\left(c_{9}(d-1) / c_{8}\right)^{-2 / d}$. Now, we observe that

$t_{\epsilon}^{-(d-1) / 2} p_{C}\left(t_{\epsilon}\right) \stackrel{(9)}{\leq} q_{\epsilon}\left(t_{\epsilon}\right)=c_{8}\left(c_{10} \epsilon^{-2 j_{0} / d}\right)^{-1 / 2}+c_{9} \epsilon^{j_{0}}\left(c_{10} \epsilon^{-2 j_{0} / d}\right)^{(d-1) / 2}=c_{11} \epsilon^{j_{0} / d}$ with $c_{11}:=c_{8} c_{10}^{-1 / 2}+c_{9} c_{10}^{(d-1) / 2}$. This implies that if $\mathfrak{b}_{N_{1, j_{0}}(\epsilon)}(K)>t_{\epsilon}$ then

$$
\mathfrak{g}_{N_{1, j_{0}}(\epsilon)}(K) \stackrel{(8)}{\leq}\left(t_{\epsilon}^{-(d-1) / 2} p_{C}\left(t_{\epsilon}\right)\right)^{2 /(d-1)} \leq\left(c_{11} \epsilon^{j_{0} / d}\right)^{2 /(d-1)} \leq \delta_{1, j_{0}} \epsilon^{\beta}
$$

with $\delta_{1, j_{0}}:=c_{11}^{2 /(d-1)}$ and $\beta:=2 j_{0}(d-1)^{-1} d^{-1}$.

It remains only to set $N_{1, j_{0}}(\epsilon)$ such that $\mathfrak{b}_{N_{1, j_{0}}(\epsilon)}(K)>t_{\epsilon}$. Set

$$
c_{12}:=\frac{\kappa_{d}}{2}+\sum_{k=1}^{d-1} \frac{(d-k) \kappa_{d-k}}{2 d}\left(\frac{v_{k}}{v_{1}}\right)^{k} \text { and } \tilde{p}(t):=c_{12} t^{d-1} .
$$


Again because of the isoperimetric inequality, we have that $p_{C}(t)<\tilde{p}(t)$, for any $t>1$. Hence if $u>\tilde{p}(1)=c_{12}$ then $p_{C}^{-1}(u)>\tilde{p}^{-1}(u)$. Set

$$
N_{1, j_{0}}(\epsilon):=c_{5} c_{12} t_{\epsilon}^{d-1}=n_{1, j_{0}} \epsilon^{-\alpha}
$$

with $n_{1, j_{0}}:=c_{5} c_{12} c_{10}^{d-1}$ and $\alpha:=2 j_{0}(d-1) d^{-1}$. Thus we have

$$
\mathfrak{b}_{N_{1, j_{0}}(\epsilon)}(K)=p_{C}^{-1}\left(c_{5}^{-1} N_{1, j_{0}}(\epsilon)\right)=p_{C}^{-1}\left(c_{12} t_{\epsilon}^{d-1}\right)>\tilde{p}^{-1}\left(c_{12} t_{\epsilon}^{d-1}\right)=t_{\epsilon}
$$

whenever $t_{\epsilon}>1$. But $t_{\epsilon}>1$ when $\epsilon<c_{10}^{-1 / \alpha}$. This completes the proof.

\section{Proof of Theorem 1.1}

Theorem 1.1 is a direct consequence of the following lemma and point 4 of Lemma 4.2. Let $c_{13}>c_{6}$.

Lemma 5.1. Let $K \in \mathcal{K}$. For any $n>c_{7}$, we have

$$
\mathfrak{d}_{1}(K, n)<c_{13} \mathfrak{g}_{n}(K) V_{1}(K) .
$$

I.e. for any integer $n>c_{7}$, there exists a polytope $P \supset K$ with $n$ facets such that

$$
\mathrm{d}_{H}(K, P)<c_{13} \mathfrak{g}_{n}(K) \frac{V_{1}(K)}{n^{2 /(d-1)}} .
$$

Proof. The condition $n>c_{7}$ implies that $\mathfrak{b}_{n}(K)$ and $\mathfrak{g}_{n}(K)$ are well defined. Let $t \in\left(0, \mathfrak{b}_{n}(K)\right)$. We have defined $\mathfrak{b}_{n}(K)$ precisely such that the convex body $t K$ and the number $n$ satisfy the conditions required to apply Theorem 1.2. So there exists a polytope $P_{t}$ with $n$ facets such that

$$
\mathrm{d}_{H}\left(t K, P_{t}\right)<c_{6} V_{d-1}\left(t C+B^{d}\right)^{2 /(d-1)} n^{-2 /(d-1)} .
$$

Therefore, for any $t \in\left(0, \mathfrak{b}_{n}(K)\right)$, we see that

$$
\mathrm{d}_{H}\left(K, \frac{1}{t} P_{t}\right)<c_{6} \frac{V_{d-1}\left(t K+B^{d}\right)^{2 /(d-1)}}{t} n^{-2 /(d-1)} .
$$

Since $c_{13}>c_{6}$, there exists $t_{0} \in\left(0, \mathfrak{b}_{n}\right)$ such that

$$
\mathrm{d}_{H}\left(K, \frac{1}{t_{0}} P_{t_{0}}\right)<c_{13}\left(\inf _{t \in\left(0, \mathfrak{b}_{n}\right)} \frac{V_{d-1}\left(t K+B^{d}\right)^{2 /(d-1)}}{t}\right) n^{-2 /(d-1)} .
$$

But it holds that

$$
\inf _{t \in\left(0, \mathfrak{b}_{n}\right)} \frac{V_{d-1}\left(t K+B^{d}\right)^{2 /(d-1)}}{t}=\mathfrak{f}_{n}(K)=\mathfrak{g}_{n}(K) V_{1}(K),
$$

which yields the proof. 


\section{References}

[1] Shiri Artstein-Avidan, Apostolos Giannopoulos, and Vitali D. Milman. Asymptotic geometric analysis. Part I, volume 202 of Mathematical Surveys and Monographs. American Mathematical Society, Providence, RI, 2015.

[2] Keith Ball. An elementary introduction to modern convex geometry. In Flavors of geometry, volume 31 of Math. Sci. Res. Inst. Publ., pages 1-58. Cambridge Univ. Press, Cambridge, 1997.

[3] K. J. Böröczky. Approximation of general smooth convex bodies. Adv. Math., 153(2):325-341, 2000.

[4] Andreas Brieden, Peter Gritzmann, Ravindran Kannan, Victor Klee, László Lovász, and Miklós Simonovits. Deterministic and randomized polynomial-time approximation of radii. Mathematika, 48(1-2):63-105 (2003), 2001.

[5] E. M. Bronstein. Approximation of convex sets by polytopes. J. Math. Sci., 153(6):727-762, 2008.

[6] E. M. Bronstein and L. D. Ivanov. The approximation of convex sets by polyhedra. Sib. Mat. J., 16(5):852-853, 1975.

[7] R. M. Dudley. Metric entropy of some classes of sets with differentiable boundaries. J. Approximation Theory, 10:227-236, 1974.

[8] L. Fejes Tóth. Approximation by polygons and polyhedra. Bull. Amer. Math. Soc., 54(4):431-438, 1948.

[9] P. M. Gruber. Aspects of approximation of convex bodies. In Handbook of convex geometry, Vol. A, pages 319-345. Elsevier, 1993.

[10] P. M. Gruber. Asymptotic estimates for best and stepwise approximation of convex bodies. I. Forum Math., 5(3):281-297, 1993.

[11] P. M. Gruber. Convex and discrete geometry, volume 336 of Grundlehren der Mathematischen Wissenschaften [Fundamental Principles of Mathematical Sciences]. Springer, Berlin, 2007.

[12] P.M. Gruber. Approximation by convex polytopes. In Polytopes: abstract, convex and computational (Scarborough, ON, 1993), volume 440 of NATO Adv. Sci. Inst. Ser. C Math. Phys. Sci., pages 173-203. Kluwer Acad. Publ., Dordrecht, 1994.

[13] Fritz John. Extremum problems with inequalities as subsidiary conditions. In Studies and Essays Presented to R. Courant on his 60th Birthday, January 8, 1948, pages 187-204. Interscience Publishers, Inc., New York, N. Y., 1948.

[14] D. E. McClure and R. A. Vitale. Polygonal approximation of plane convex bodies. J. Math. Anal. Appl., 51(2):326-358, 1975.

[15] S. Reisner, C. Schütt, and E. Werner. Dropping a vertex or a facet from a convex polytope. Forum Math., 13(3):359-378, 2001. 
[16] Ross M. Richardson, Van H. Vu, and Lei Wu. An inscribing model for random polytopes. Discrete Comput. Geom., 39(1-3):469-499, 2008.

[17] R. Schneider. Zur optimalen Approximation konvexer Hyperflächen durch Polyeder, volume $=256$, year $=1981$, doi $=10.1007 /$ BF01679698, issn $=0025-5831$, url = http://link.springer.com/10.1007/BF01679698, Math. Ann., (3):289-301.

[18] R. Schneider. Polyhedral approximation of smooth convex bodies. J. Math. Anal. Appl., 128(2):470-474, 1987. 